\title{
Refinement of Crystal Structural Parameters and Charge Density using Convergent-Beam Electron Diffraction
}

\author{
K. Tsuda, Y. Ogata and M. Tanaka
}

Institute of Multidisciplinary Research for Advanced Materials, Tohoku University, Sendai 980-8577, JAPAN

We have developed a new method to refine crystal structural parameters using convergent-beam electron diffraction [1]. The method is based on the fitting between theoretical calculations and experimental intensities of energy-filtered two-dimensional CBED patterns containing both of zeroth-order Laue-zone (ZOLZ) reflections and higher-order Laue-zone (HOLZ) reflections. The use of HOLZ reflections is essential for the present method because small displacements of atoms can be sensitively detected using HOLZ reflections with large reciprocal vectors. For this purpose, we developed an Omega-filter transmission microscope JEM-2010FEF [1], [2], which can take energy-filtered CBED patterns up to a high angle to include HOLZ reflections with a small distortion, and an analysis program MBFIT to refine structural parameters, which is based on many-beam Bloch-wave calculations and nonlinear least-squares fitting. Using the method, we refined the atom positions and anisotropyic Debye-Waller factors of the rhombohedral phase of $\mathrm{LaCrO}_{3}$, which is a perovskite-type material for interconnector of solid oxide fuel cells. Clear anisotropy of the thermal vibration of the oxygen atoms was successfully detected for the first time [3].

The present method can be applied to the determination of charge density distribution because the low-order Fourier coefficients of the electrostatic potential (low-order crystal structure factors for electron diffraction), which are sensitive to valence electrons, can be refined together with the atom positions and Debye-Waller factors. Through Poisson's equation, the structure factors for electron diffraction are related to those for X-ray diffraction, or the Fourier coefficients of the charge density. According to the nature of Poisson's equation, a small change in the low-order structure factors for $\mathrm{X}$-rays causes a large change in those for electrons. It should be noted that the accurate determination of Debye-Waller factors, which can be performed successfully by the present method, is crucial to obtaining high-precision low-order structure factors for X-rays from the structure factors for electrons. Figure 2(a) shows the charge density of the $\mathrm{CrO}_{2}$ plane determined by the present method. Figure 2(b) shows the deformation charge density of the $\mathrm{CrO}_{2}$ plane, or the difference between the charge density of Fig. (a) and the charge density calculated with the neutral atoms. The charge transfer from the $\mathrm{Cr}$ atoms to the $\mathrm{O}$ atoms is clearly seen.

The anisotropic charge density of the orbital-ordering phase of $\mathrm{LaMnO}_{3}$ determined by the present method will be also presented in the conference.

\section{References}

[1] K. Tsuda and M. Tanaka, Acta Cryst., A55 (1999) 939.

[2] M. Tanaka et al., J. Microsc., 194 (1999) 219.

[3] K. Tsuda and M. Tanaka, Microsc. Microanal., 6 (Suppl. 2) (2000) 152. 


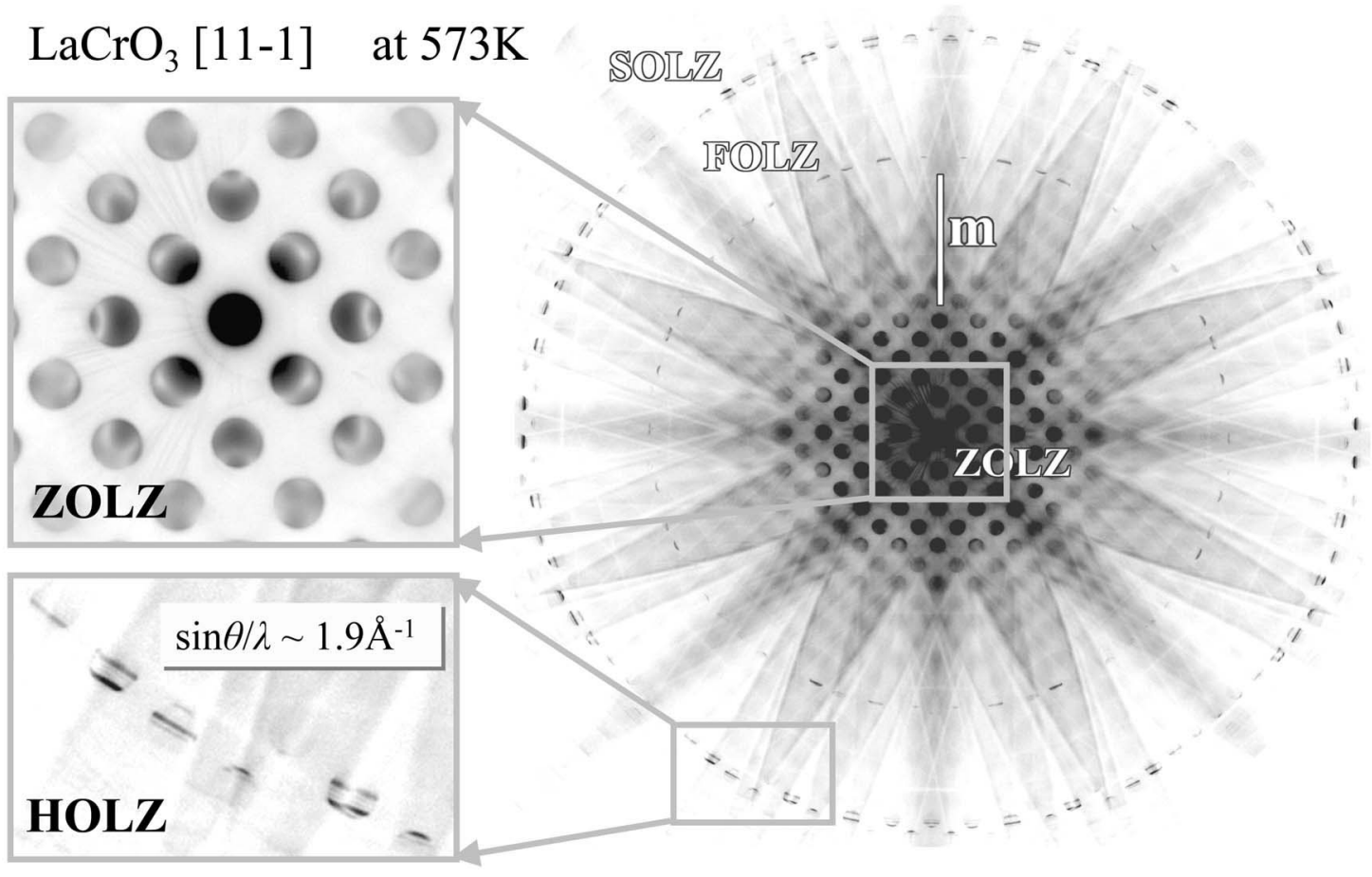

Fig. 1: An energy-filtered CBED pattern of the rhombohedral phase of $\mathrm{LaCrO}_{3}$. The HOLZ reflections with large reciprocal vectors $\left(\sin \theta / \lambda \sim 1.9 \AA^{-1}\right)$ are seen.

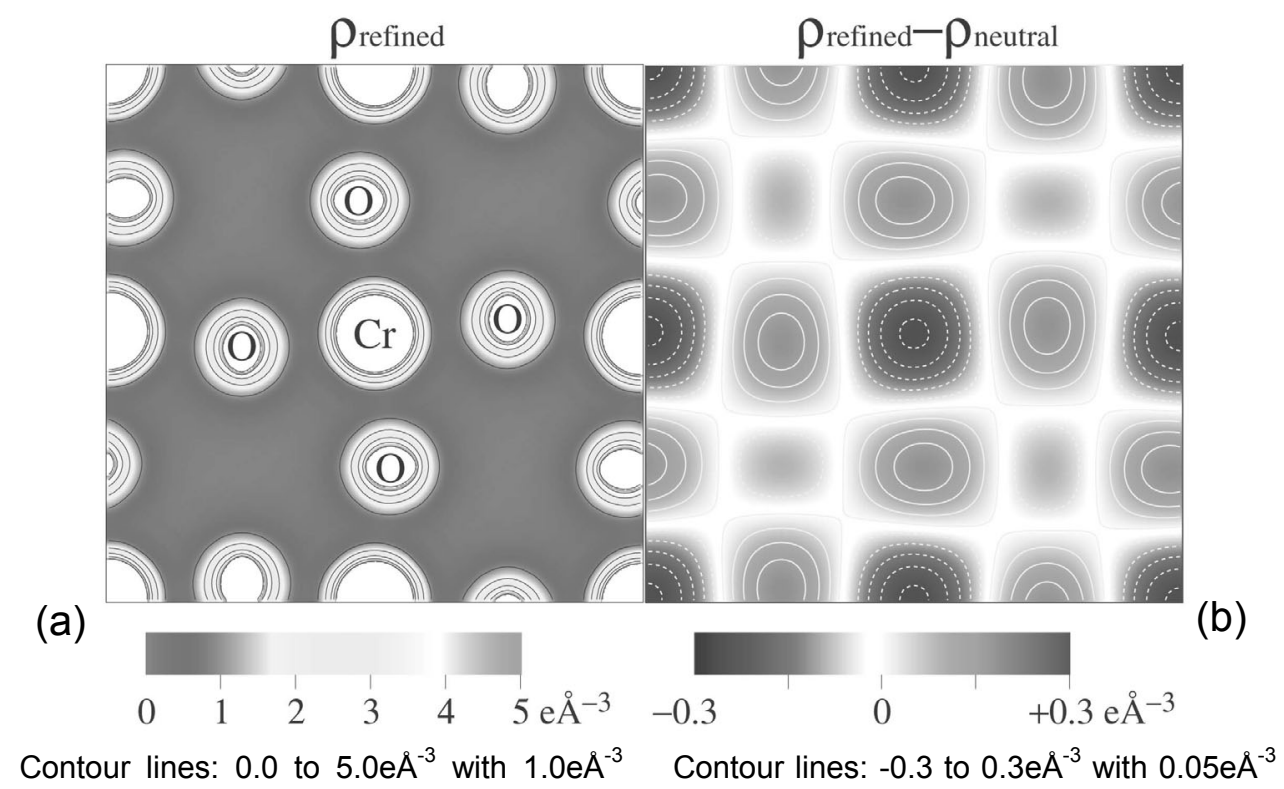

Fig. 2: Charge density (a) and deformation charge density (b) at the $\mathrm{CrO}_{2}$ plane determined by the present method. The solid lines and dotted lines of the contour maps indicate positive and negative values, respectively. 